PROGRAMA DE INICIAÇÃO CIENTÍFICA 


\section{Unce $=\mathbf{B}$ \\ Centro Unıversitárıo de Brasílıa}

FABIANA TAVARES PEREIRA

\section{CARACTERIZAÇÃO DO ATENDIMENTO PSICOLÓGICO EM ENFERMARIA PEDIÁTRICA}

Relatório final de pesquisa de Iniciação Científica apresentado à Assessoria de Pós-Graduação e Pesquisa pela Faculdade de Ciências da

Educação e Saúde - FACES.

Orientação: Marina Kohlsdorf

\section{BRASÍLIA}

2017 


\title{
CARACTERIZAÇÃO DO ATENDIMENTO PSICOLÓGICO EM ENFERMARIA PEDIÁTRICA
}

\author{
Fabiana Tavares Pereira - UniCEUB, PIC voluntário \\ fabitavares.p@gmail.com
}

\section{Marina Kohlsdorf - UniCEUB, professora orientadora marina.kohlsdorf@uniceub.br}

Adentrando o campo hospitalar, o olhar para o profissional de psicologia que atua na área de pediatria tem se mostrado fundamental para obtenção de bons resultados com crianças e cuidadores em face aos mais variados tratamentos, especialmente devido às características psicossociais que permeiam as principais demandas em saúde da população pediátrica brasileira. Estes aspectos nortearam o objetivo geral do presente estudo, que buscou traçar um perfil sociodemográfico e psicossocial dos pacientes atendidos na enfermaria pediátrica do Hospital Materno-Infantil de Brasília se valendo de uma análise documental dos prontuários da unidade. Os dados obtidos possibilitaram observar a relação estabelecida pelo contexto social que insere os pacientes, assim como as características dos diagnósticos e de outros aspectos associados (e.g.: dinâmica familiar) que mostraram influenciar na condição psicológica identificada nos casos acompanhados, em específico no que se refere ao enfrentamento das condições. Pensando na atuação do profissional de psicologia na equipe multidisciplinar, houve indícios de uma rotina de trabalho que prioriza o contato com o público atendido e a comunicação entre os membros da equipe sobre os acompanhamentos realizados em detrimento ao registro documental (prontuários preenchidos parcialmente, porém com informações sobressalentes). O estudo apontou para a necessidade da ampliação das redes de apoio que realizem um trabalho conjunto com a hospitalar (pensando o hospital como um ponto de matriciamento), visto a quantidade de casos interseccionados por questões socioeconômicas e de vulnerabilidade social. Além disto, contribuiu com dados estatísticos e subjetivos que podem auxiliar em intervenções e estratégias futuras no campo da psicologia saúde, não apenas pediátrica.

Palavras-Chave: Psicologia da saúde. Enfermaria pediátrica. Psicologia pediátrica. Atendimento psicológico. 


\section{Introdução}

A melhoria à assistência pediátrica ocorrida nos últimos 50 anos promoveu melhores condições de vida aos pacientes e também o destaque à necessidade de atendimento em equipes interdisciplinares. Desta forma, houve o surgimento de uma área específica, a Psicologia Pediátrica, para aprofundar as ações preventivas, promotoras à saúde e interventivas (Viana \& Almeida, 1998).

Na população pediátrica brasileira, demandas em saúde se caracterizam de forma marcante também por questões psicossociais, tais como ansiedade pela resposta clínica, dificuldades na dinâmica familiar, separação geográfica da família, demandas profissionais e financeiras, sobrecarga de cuidados parentais, adesão aos cuidados, comunicação com a equipe e enfrentamento do diagnóstico e seus desdobramentos (Armelin et. al., 2005; Beltrão, Vasconcelos, Pontes \& Albuquerque, 2007; Brown \& Wissow, 2008; \& Savage, 2010). Este fato ressalta a necessidade de se conhecer como o profissional em psicologia tem atuado junto a pacientes pediátricos e seus familiares de forma sistemática e objetiva, a fim de subsidiar ações mais eficientes no cuidado à população pediátrica.

A literatura advoga que intervenções por parte da equipe de psicologia em contexto pediátrico têm contribuído de forma fundamental para menos retornos ambulatoriais, maior satisfação do usuário com o serviço, melhor adesão aos cuidados, melhor qualidade de vida, menores perturbações psicológicas relacionadas a ansiedade, depressão e estresse pós-traumático, menor sobrecarga de trabalho para a equipe e maior colaboração de crianças e cuidadores com procedimentos invasivos (Clark et al., 2000; Coad, 2007; Costa Junior, 2001; 2005; Flury et. al., 2011; Nobile \& Drotar, 2003; Oliveira \& Gomes, 2004). Além disso, o acompanhamento psicológico prestado à criança e seus acompanhantes durante um episódio de adoecimento é pressuposto essencial do processo de humanização em saúde (Brasil, 2004; Deslandes, 2004; Lei n. 8.080, 1990).

\section{Objetivos}

Constitui objetivo geral deste estudo realizar uma análise retrospectiva de dados documentais de prontuários para caracterizar o perfil socidemográfico e 
psicossocial de pacientes atendidos na enfermaria pediátrica do Hospital MaternoInfantil de Brasília e as ações desenvolvidas pela equipe de psicologia, intuindo especificamente:

1) Caracterizar quantitativamente 0 perfil sociodemográfico e epidemiológico dos pacientes atendidos na enfermaria pediátrica do Hospital Materno-Infantil de Brasília;

2) Descrever e caracterizar de forma quantitativa a atuação da equipe de psicologia junto a pacientes e seus familiares;

3) Investigar associações entre as variáveis sociodemográficas, demandas psicossociais e intervenções em âmbito psicológico, a partir de análises estatísticas inferenciais multivariadas.

\section{Fundamentação teórica}

A psicologia da saúde consiste na busca pela compreensão dos processos relativos ao fenômeno saúde-doença, considerando o modelo biopsicossocial para sua aplicação e ampliando a visão acerca do conceito de saúde - que passa a ser entendido como presença de bem-estar e não ausência de doença -, considerando questões físicas e mentais para avaliação, diagnóstico, tratamento e prevenção dos casos acompanhados. Engloba aspectos que excedem o contexto hospitalar, preocupando-se com todo 0 ambiente que circunscreve o paciente e seus cuidadores, aceitando que o social impacta diretamente nas intervenções necessárias a serem realizadas (Castro \& Bornholdt, 2004; Castro \& Piccinini, 2002; Costa Junior, 2005; Daneluci, 2013; Spink, 1992; Straub, 2014a).

Dentro da perspectiva trazida pela psicologia da saúde, observou-se desde cedo que a área de pediatria pedia uma atenção especial por parte dos psicólogos, pois características específicas no atendimento infantil se associavam aos processos psicológicos e de desenvolvimento que excediam o modelo biomédico, havendo a possibilidade frequente da interlocução entre a psicologia clínica infantil e a pediatria, ao contrário do que ocorria com os adultos. Com o advento de novas especialidades pediátricas na medicina a emergência da psicologia pediátrica ficou evidente, trazendo colaboração efetiva para a prevenção e tratamento de doenças agudas e crônicas (Barros, 1999; Castro, 2007; Viana \& Almeida, 1998). 
Barros (1999), Castro (2007) e Viana \& Almeida (1998) definem a psicologia pediátrica como um campo de atuação interdisciplinar, que intervém em questões físicas e mentais do fenômeno de saúde-doença junto às crianças e adolescente adoecidos em comunhão com seus cuidadores, considerando aspectos ambientais, cognitivos, emocionais e sociais que possam interferir - ou que estejam interferindo - no diagnóstico e tratamento do quadro. A preocupação com a promoção de bemestar, preservação do desenvolvimento físico-cognitivo (dentro das possibilidades) e a prevenção de doenças e acidentes também é constituinte da área.

É importante salientar que a intervenção junto aos cuidadores configura um ponto central da atuação do psicólogo da saúde que atua em pediatria, visto que estes têm sua responsabilidade estendida ao lidar com as próprias demandas emocionais, mediar as respostas do núcleo familiar e manter uma comunicação coerente com todas as partes envolvidas - o que inclui os profissionais de saúde (Kohlsforf \& Costa Júnior, 2012).

A rotina enfrentada pelos pacientes pediátricos e seus familiares submete-os a situações estressoras, uma vez que os indivíduos percebem que a situação acarreta em prejuízos físicos e emocionais que interferem em seu bem-estar. Este acontecimento justifica a massiva produção literária sobre a temática de adoecimento crônico infantil abordando o enfrentamento (coping), conceito referente às medidas comportamentais que os indivíduos utilizam para lidar com o evento estressor se utilizando de seus recursos pessoais (Castro \& Piccinini, 2002; Flury et. al., 2011; Mensorio, et. al., 2009; Kohlsdorf \& Costa Junior, 2012; Pereira \& Araújo, 2005; Straub, 2014b).

Kohlsdorf e Costa Junior (2012) realizaram uma revisão de literatura que apontou uma produção bibliográfica extensa sobre os aspectos psicossociais do câncer infantil para os pais, a qual demonstrou que 14\% dos estudos estão centrados nas perturbações psicológicas acarretadas pelo enfrentamento da situação; $12 \%$ discorrem sobre o impacto para a dinâmica familiar; $18 \%$ trazem à tona a necessidade de ajustamento psicossocial; e $23 \%$ tratam sobre as dificuldades, demandas e recursos que advém com o quadro. Os números transparecem a urgência e a importância de se considerar os cuidadores durante 
todo o processo de acompanhamento psicológico do caso, pois o adoecimento crônico infanto-juvenil acomete toda a rede de suporte familiar.

Devido a este comprometimento da relação familiar, para o manejo dos casos de adoecimento crônico e agudo pediátrico, a atuação do profissional de psicologia deve considerar todas as partes envolvidas no caso (paciente, familiares e equipe de saúde) com o objetivo de garantir uma melhor comunicação interpessoal, visto que este processo demanda 0 quanto 0 paciente compreende $\mathrm{e}$ adere às recomendações que lhe são transmitidas, sendo essencial que as informações sejam passadas de modo esclarecedor e inteligível ao ouvinte. É por meio desta atuação multidisciplinar e multideterminada enfocando a comunicação em saúde que o psicólogo auxilia na adesão aos cuidados, que diz respeito ao quanto os indivíduos - paciente e familiares - seguem as recomendações médicas (Armelin et. al., 2005; Coutinho, 2013; Nobile \& Drotar, 2003; Oliveira \& Gomes, 2004).

Desse modo, conhecer os aspectos sociais e o contexto geral que insere as famílias se apresenta como um dado essencial para auxiliar o tratamento do adoecimento crônico infanto-juvenil de forma mais completa e integrativa. No entanto, não há estudos relacionando e elencando dados sociodemográficos, psicossociais e epidemiológicos na região do Distrito Federal e entorno, o que reitera a importância da pesquisa em questão.

\section{Metodologia}

\subsection{Participantes}

Crianças que estiveram internadas na enfermaria pediátrica do Hospital Materno-Infantil de Brasília e seus cuidadores durante o ano de 2015. Foram analisados 353 casos com dados registrados em prontuários de atendimento psicológico.

\subsection{Material, Instrumento e Local}

A coleta de dados foi realizada a partir de registros por escrito nos protocolos de acolhimento e acompanhamento utilizados no atendimento de crianças hospitalizadas em uma enfermaria específica no Hospital Materno-Infantil de Brasília. Tais fichas correspondem ao prontuário psicológico da criança, são 
preenchidas ao longo do atendimento e permanecem em local guardado por chave, ao qual somente a psicóloga responsável pelo serviço tem acesso.

Nestas fichas, os seguintes dados são registrados: (1) Dados psicossociais da criança e de sua família (idade, procedência, constituição familiar, endereço, entre outros); (2) Dados da enfermidade (diagnóstico, tempo de tratamento, número de internações, cuidador principal, entre outros); e (3) Tipo de atendimento psicológico prestado e seu desenvolvimento ao longo da internação (ronda, avaliação psicológica, procedimentos de preparação cirúrgica ou para procedimentos invasivos, encaminhamentos a outros serviços, orientação sobre práticas educativas parentais, estimulação precoce, entre outros).

\subsection{Procedimento}

Este projeto se insere neste contexto de atendimento psicológico a crianças e seus familiares acolhidos no HMIB durante o ano de 2015, especificamente em uma enfermaria pediátrica que recebe crianças com adoecimentos agudos (asma, bronquite e pneumonia em sua maioria) e também crônicos (má formação cardíaca, cirurgia pediátrica, transplantes cardíacos, anemia falciforme e distúrbios metabólicos). O projeto de pesquisa foi inicialmente submetido à apreciação ética pelo Comitê de Ética em Pesquisa da Fundação de Ensino e Pesquisa em Ciências da Saúde (FEPECS), tendo sido aprovado em 14 de março de 2017 sob protocolo 1.963.203.

Trata-se de um estudo descritivo e exploratório acerca das intervenções psicológicas realizadas junto aos 353 pacientes internados durante o ano de $2015 \mathrm{e}$ seus familiares em três alas distintas, como parte do trabalho desenvolvido pela professora orientadora da pesquisa, que atua em tempo parcial como psicóloga no referido hospital. Os atendimentos psicológicos são registrados por escrito em um formulário desenvolvido especificamente para este acompanhamento, que inclui uma caracterização sociodemográfica da criança e de sua família e um diário discursivo sobre as intervenções psicossociais realizadas durante o período de internação da criança. Tal formulário sofreu remodelações para ampliar as informações coletadas e permitir melhor conhecimento dos fatores envolvidos, de modo que alguns dos prontuários analisados não continham todas as questões avaliadas devido a estas reformulações, contabilizando um total de cinco versões 
diferentes contempladas pela pesquisa. Além disto, haviam alguns prontuários que estavam com informações em branco.

Os dados sociodemográficos registrados no protocolo incluem: idade da criança, de seus irmãos e de seus cuidadores, número de filhos na família, escolaridade da criança, irmãos e cuidadores, estado civil dos pais, procedência, renda familiar mensal, diagnóstico primário da internação, quantidade de dias de internação, comorbidades físicas, breve história da saúde e adoecimento da criança. O diário discursivo sobre $\mathrm{O}$ atendimento psicológico aborda as técnicas e procedimentos empregados durante a hospitalização (por exemplo, escuta ativa, dissonância cognitiva, técnicas para relaxamento, diário de automonitoramento, entre outros), investigação sobre a dinâmica familiar e estilo de práticas educativas parentais, demandas psicossociais identificadas durante a hospitalização (tais como presença de violência em suas diversas modalidades ou riscos ao desenvolvimento afetivo saudável da criança, necessidade de encaminhamentos a Conselho Tutelar ou Centros de Referência em Assistência Social - CRAS), processos básicos relacionados à internação (enfrentamento, adesão aos cuidados, comunicação com a equipe de saúde, presença e qualidade da rede de suporte sociofamiliar, dificuldades vivenciadas durante a internação, manifestações de ansiedade, depressão, estresse pós-traumático, entre outros) e demais assuntos relacionados à prática do psicólogo neste contexto de intervenção.

A análise dos dados utilizou metodologia mista, em que os dados contidos nos prontuários foram incluídos em um banco de dados no excel e submetidos a análises de estatística descritiva e inferencial multivariadas, conforme proposto por Dancey \& Reidy (2011). Com os dados devidamente circunscritos na plataforma digital, uma visão do panorama geral dos atendimentos possibilitou também observar categorias nas quais os casos poderiam ser agrupados de acordo com suas semelhanças, o que permitiu a aplicação dos pressupostos de Bardin (1977) para uma análise qualitativa do conteúdo obtido.

\section{Resultados/Discussão}

Durante a exploração dos prontuários foi possível identificar diferenças nos preenchimentos que não se restringiam aos variados formatos e sim aos profissionais responsáveis: haviam diagnósticos semelhantes cujas informações sobressalentes diferiam. No entanto, a principal característica observada, e que teve 
interferência nos resultados do estudo, diz respeito aos campos que eram deixados em branco, de modo que não era possível acessar todos os aspectos do caso que deveriam constar nos formulários.

Em contrapartida, haviam anotações que extrapolavam as perguntas prédeterminadas e que auxiliaram na definição da condição psicológica dos atendidos na enfermaria. Essa disparidade aferida nos preenchimentos apresentou indícios de uma atuação que prioriza o contato com o público atendido e a troca de informações entre os membros da equipe que excede o registro documental, sendo este apenas um instrumento de apoio para a condução dos acompanhamentos. A Tabela 1 a seguir apresenta os dados descritivos dos 353 casos estudados: 
Tabela 1: Características dos pacientes e cuidadores

\begin{tabular}{|c|c|}
\hline Características dos pacientes e cuidadores & Frequências \\
\hline \multicolumn{2}{|l|}{ Sexo } \\
\hline Feminino & 174 \\
\hline masculino & 165 \\
\hline \multicolumn{2}{|l|}{ Idade dos pacientes } \\
\hline \multicolumn{2}{|l|}{ Média 31,73 meses (2,5 anos) } \\
\hline \multicolumn{2}{|l|}{ Desvio-Padrão 38,98 meses (3 anos) } \\
\hline \multicolumn{2}{|l|}{ Mínimo recém nascido } \\
\hline \multicolumn{2}{|l|}{ Máximo 204 meses (17 anos) } \\
\hline \multicolumn{2}{|l|}{ Estado Civil dos cuidadores } \\
\hline Solteiros ou divorciados & 80 \\
\hline Casados ou união estável & 142 \\
\hline \multicolumn{2}{|l|}{ História de hospitalizações } \\
\hline Primeira hospitalização da criança & 94 \\
\hline Internações anteriores & 133 \\
\hline \multicolumn{2}{|l|}{ Duração da internação atual } \\
\hline Até uma semana & 216 \\
\hline Mais de uma semana & 108 \\
\hline Indicação cirúrgica & 11 \\
\hline \multicolumn{2}{|l|}{ Diagnóstico principal } \\
\hline Tetralogia de Fallot & 9 \\
\hline Pneumonia & 134 \\
\hline Bronquiolite & 34 \\
\hline Síndromes cromossômicas diversas & 33 \\
\hline Febre não especificada & 13 \\
\hline Outras cardiopatias & 60 \\
\hline Atresia pulmonar & 5 \\
\hline Asma & 36 \\
\hline Bronquite & 17 \\
\hline $\begin{array}{l}\text { Outros (ingestão de chumbinho, anemia falciforme, febre reumática, fratura, infecção } \\
\text { urinária, alergia alimentar) }\end{array}$ & 12 \\
\hline \multicolumn{2}{|l|}{ Prognóstico } \\
\hline Bom & 125 \\
\hline Adverso & 89 \\
\hline \multicolumn{2}{|l|}{ Suporte social: recebe visitas } \\
\hline Sim & 113 \\
\hline Não & 34 \\
\hline \multicolumn{2}{|l|}{ Suporte Social: alterna presença na internação com outros cuidadores } \\
\hline Sim & 46 \\
\hline Não & 103 \\
\hline \multicolumn{2}{|l|}{ Queixas físicas do cuidador } \\
\hline Sim & 54 \\
\hline Não & 89 \\
\hline \multicolumn{2}{|l|}{ Queixas emocionais do cuidador } \\
\hline Sim & 68 \\
\hline Não & 69 \\
\hline \multicolumn{2}{|l|}{ Queixas do cuidador sobre rotinas do hospital } \\
\hline $\operatorname{Sim}$ & 33 \\
\hline Não & 113 \\
\hline \multicolumn{2}{|l|}{ Queixas do cuidador sobre equipe do hospital } \\
\hline Sim & 20 \\
\hline Não & 126 \\
\hline \multicolumn{2}{|l|}{ Déficit cognitivo do paciente } \\
\hline Sim & 25 \\
\hline Não & 196 \\
\hline \multicolumn{2}{|l|}{ Comportamento agressivo do paciente } \\
\hline Sim & 22 \\
\hline Não & 201 \\
\hline
\end{tabular}


De forma geral, destaca-se na Tabela 1 minoria de casos indicando queixas físicas, emocionais ou relacionadas à estadia no hospital. $O$ alto número de cuidadores que relataram receber visitas também constitui aspecto importante, tendo em vista que o suporte social pode auxiliar a adaptação de familiares ao contexto de internação pediátrica (Castro \& Piccinini, 2002; Flury et. al., 2011; Mensorio, et. al., 2009; Kohlsdorf \& Costa Junior, 2012; Pereira \& Araújo, 2005; Straub, 2014b). Por fim, torna-se relevante destacar a quantidade de diferentes diagnósticos acompanhados, que se caracterizam por condições agudas (febre, pneumonia, bronquite) até contexto crônicos bastante complexos (cardiopatias, síndromes cromossômicas).

Foram realizadas análises estatísticas inferenciais para verificar associações entre as condições psicológicas dos cuidadores (queixas físicas, emocionais, sobre o contexto de internação) e dados sociodemográficos. Apenas os resultados estatisticamente significativos serão descritos a seguir.

Relatos de agressividade por parte do paciente foram associados a diversas variáveis: queixas de cuidadores quanto à equipe de saúde $\left(X^{2}=143,68 ; p<0,001\right)$; queixas físicas dos cuidadores $\left(X^{2}=8,56 ; p<0,003\right)$, queixas dos cuidadores sobre a rotina do hospital $\left(X^{2}=43,83 ; p<0,001\right)$. Estes dados destacam o quanto uma internação pode prejudicar aspectos relacionais que os cuidadores estabelecem com seus filhos e com a equipe, enfatizando a importância da atuaçaõ do psicólogo como mediador neste contexto (Armelin et. al., 2005; Coutinho, 2013; Nobile \& Drotar, 2003; Oliveira \& Gomes, 2004).

A importância do suporte social também foi um resultado enfatizado nos dados obtidos. Cuidadores que se revezavam nos cuidados apresentaram menos queixas físicas $\left(X^{2}=8,56 ; p=0,003\right)$ e queixas quanto à equipe de saúde $\left(X^{2}=76,95\right.$; $p<0,001)$.

Por outro lado, elementos específicos do adoecimento se mostraram bastante relevantes em relação à saúde física e psicológica dos cuidadores. Acompanhantes vivenciando sua primeira internação pediátrica indicaram mais queixas físicas $\left(X^{2}=8,82 ; p<0,001\right)$ e emocionais $\left(X^{2}=43,87 ; p<0,001\right)$. Prognósticos reservados estiveram também associados a queixas sobre a rotina hospitalar $\left(X^{2}=132,43\right.$; $p<0,001)$ e sintomas físicos $\left(X^{2}=21,80 ; p<0,001\right)$. Esses dados em conjunto oferecem suporte ao que a literatura advoga, em que o contexto psicossocial e a vivência do tratamento pediátrico se encontram intimamente relacionados (Castro \& 
Bornholdt, 2004; Castro \& Piccinini, 2002; Costa Junior, 2005; Daneluci, 2013; Spink, 1992; Straub, 2014a).

\section{Considerações finais}

Este estudo investigou associações entre elementos relacionados à vivência da internação pediátrica por cuidadores, incluindo aspectos demográficos, clínicos e queixas diversas relacionadas ao processo de hospitalização. Os dados destacam a importância da atuação de equipes de saúde mental no suporte a cuidadores e pacientes durante este contexto adverso. Além disso, destaca-se ainda a necessidade de ampliação na rede de matriciamento, de modo a oferecer aos familiares de crianças internadas o devido suporte operacional e emocional tão necessário na vivência de uma internação. Ações pautadas em Políticas Públicas se fazem necessárias para ampliar as possibilidades de atuação do psicólogo neste contexto.

\section{Referências Bibliográficas}

Armelin, C.B., Wallau, R.A., Sarti, C.A. \& Pereira, S.R. (2005). A comunicação entre os profissionais de pediatria e a criança hospitalizada. Revista Brasileira de Crescimento e Desenvolvimento Humano, 15(2), 45-54.

Bardin, L. (2011/1977). Análise de Conteúdo. Lisboa: Editora 70.

Barros, L. (1999). A Psicologia pediátrica: objecto, fundamentos e modelos. Psicologia: Teoria, Investigação e Prática, 2, 179-196.

Brasil (2004). Ministério da Saúde. Secretaria-Executiva. Núcleo Técnico da Política Nacional de Humanização - HumanizaSUS: política nacional de humanização. Brasília: Ministério da Saúde.

Brown, J.D. \& Wissow, L.S. (2008). Discussion of maternal stress during pediatric primary care visits. Ambulatory Pediatrics, 8(6), 368-374.

Buckley, A. \& Savage, E. (2010). Preoperative information needs of children undergoing tonsillectomy. Journal of Clinical Nursing, 19, 2879-2887.

Castro, E. K. de, \& Bornholdt, E. (2004). Psicologia da saúde x psicologia hospitalar: definições e possibilidades de inserção profissional. Psicologia: Ciência e Profissão, 24(3), 48-57 
Castro, E. K. de, \& Piccinini, C. A. (2002). Implicações da doença orgânica crônica na infância para as relações familiares: algumas questões teóricas. Psicologia: Reflexão e Crítica, 15(3), 625-635

Clark, N.M., Gong, M., Schork, M.A., Kaciroti, N., Evans, D., Roloff, D., Hurwitz, M., Maiman, L.A. \& Mellins, R.B. (2000). Long-term effects of asthma education for physicians on patient satisfaction and use of health services. European Respiratory Journal, 16(1), 15-21.

Coad, J. (2007). Using art-based techniques in engaging children and young people in health care consultations and/or research. Journal of Research in Nursing, 12(5), 487-497.

Costa Junior, A.L. (2001). Análise de comportamentos de crianças expostas à punção venosa para quimioterapia. (Tese de Doutorado). Universidade de Brasília, Brasília.

Costa Junior, A.L. (2005). Psicologia da Saúde e desenvolvimento humano: o estudo do enfrentamento em crianças com câncer e expostas a procedimentos médicos invasivos. Em M.A. Dessen \& A.L. Costa Junior (Orgs.), A ciência do desenvolvimento humano: tendências atuais e perspectivas futuras (171-189). Porto Alegre: Artmed.

Coutinho, S.M.G. (2013). Comunicação médico-paciente em onco-hematologia pediátrica: efeito de um procedimento de intervenção psicológica. Tese de Doutorado. Universidade de Brasília, Brasília, DF.

Dancey, C.P. \& Reidy, J. (2013). Estatística sem matemática para psicologia. Porto Alegre: Artmed.

Daneluci, R. C. (2013). Psicologia e saúde como campo de interrogações. Revista Psicologia e Saúde, 5(1), 18-24.

Deslandes, S.F. (2004). Análise do discurso oficial sobre a humanização da assistência hospitalar. Ciência \& Saúde Coletiva, 9(1), 7-14.

Flury, M., Caflisch, U., Ullmann-Bremi, A. \& Spichiger, E. (2011). Experiences of parents with caring for their child after a cancer diagnosis. Journal of Pediatric Oncology Nursing, 20(10), 1-11.

Kohlsdorf, M., Costa Junior, A. L. (2012). Impacto psicossocial do câncer pediátrico para pais: revisão da literatura. Paidéia (Ribeirão Preto), 22(51), 119-129.

Lei n. 8.080 , de 19 de setembro de 1990. Dispõe sobre as condições para promoção, proteção e recuperação da saúde, a organização e o funcionamento dos serviços correspondentes e dá outras providências. Presidência da República. Brasília, DF. 
Mensorio, M. S.; Kohlsdorf, M.; Costa Junior, A. L. (2009). Cuidadores de crianças e adolescentes com leucemia: análise de estratégias de enfrentamento. Psicologia em Revista (Belo Horizonte), Belo Horizonte, 15 (1), p. 158-17.

Nobile, C. \& Drotar, D. (2003). Research on the quality of parent-provider communication in pediatric care: Implications and recommendations. Developmental and Behavioral Pediatrics, 24(4), 279-290.

Oliveira, V.Z. \& Gomes, W.B. (2004). Comunicação médico-paciente e adesão ao tratamento em adolescentes portadores de doenças orgânicas crônicas. Estudos de Psicologia, 9(3), 459-469.

Pereira, M. E. M. S. M., \& Araujo, T. C. C. F. de. (2005). Estratégias de enfrentamento na reabilitação do traumatismo raquimedular. Arquivos de NeuroPsiquiatria, 63(2b), 502-507.

Spink, M. J. P. (1992). Psicologia da saúde: a estruturação de um novo campo de sabe. Em: Campos, F. C. B. Psicologia e saúde: repensando práticas. Säo Paulo, HUCITEC, 1992. p.11-23.

Straub, R. O. (2014). Introdução à psicologia da saúde. Em: Psicologia da saúde: uma abordagem biopsicossocial. 3 ed. Porto Alegre: Artmed.

Straub, R. O. (2014). Estresse e saúde. Em: Psicologia da saúde: uma abordagem biopsicossocial. 3 ed. Porto Alegre: Artmed.

Viana, V. \& Almeida, J.P. (1998). Psicologia pediátrica: do comportamento à saúde infantil. Análise Psicológica, 1(XVI), 29-40. 